\title{
Gene-expression signature functional annotation of breast cancer tumours in function of age
}

Pascal Jézéquel ${ }^{1,2,3,8^{*}}$, Zein Sharif ${ }^{4}$, Hamza Lasla ${ }^{1}$, Wilfried Gouraud ${ }^{1,2,3}$, Catherine Guérin-Charbonnel ${ }^{1,2,3}$, Loïc Campion ${ }^{3,5}$, Stéphane Chrétien ${ }^{6}$ and Mario Campone $e^{1,3,7}$

\begin{abstract}
Background: Breast cancer biological characteristics change as age advances. Today, there is a lack of knowledge regarding age-specific molecular alterations that characterize breast tumours, notably in elderly patients. The vast majority of studies that aimed at exploring breast cancer in function of age are based on clinico-pathological data. Gene-expression signatures (GES), which in some ways capture biological information in a non-reductionist manner, represent powerful tools able to explore tumour heterogeneity.

Methods: Twenty-five GES were used for functional annotation of breast tumours in function of age: five for molecular subtyping, seven for immune response, three for metabolism, seven for critical pathways in cancer and three for prognosis. Affymetrix ${ }^{\circledR}$ genomics datasets were exclusively used to avoid cross-platform normalization issues. Available corresponding clinico-pathological data were also retrieved and analysed.
\end{abstract}

Results: Fifteen publicly available datasets were pooled for a total of 2378 breast cancer patients (whole cohort), out of whom 1413 were of Caucasian origin. Three age groups were defined: $\leq 40$ years (AG1), $>40$ to $<70$ years (AG2) and $\geq 70$ years (AG3). We confirmed that age influenced the incidence of molecular subtypes. We found a significant growing incidence of luminal $B$ and a decreasing kinetics for basal-like in function of age. We showed that AG3 luminal B tumours were less aggressive than AG1 luminal B tumours based on different GES (iron metabolism, mitochondrial oxidative phosphorylation and reactive stroma), recurrence score prognostic GES and histological grade (SBR). Contrary to tumours of young patients, tumours of elderly patients concentrated favourable GES scores: high oestrogen receptor and mitochondrial oxidative phosphorylation, low proliferation, basal-like, glycolysis, chromosomal instability and iron metabolism, and low GES prognostic scores (van't Veer 70-GES, genomic grade index and recurrence score).

Conclusions: Functional annotation of breast tumours by means of 25 GES demonstrated a decreasing aggressiveness of breast tumours in function of age. This strategy, which can be strengthened by increasing the number of representative GES to gain more insight into biological systems involved in this disease, provides a framework to develop rational therapeutic strategies in function of age.

Keywords: Age, Breast cancer, Gene-expression signatures, Genomics

\footnotetext{
* Correspondence: pascal.jezequel@ico.unicancer.fr

${ }^{1}$ Bioinfomics unit, Integrated Centre for Oncology - René Gauducheau, Bd J.

Monod, Nantes, Saint Herblain Cedex 44805, France

${ }^{2}$ Cancer Genomic Unit, Integrated Centre for Oncology - René Gauducheau,

Bd J. Monod, Nantes, Saint Herblain Cedex 44805, France

Full list of author information is available at the end of the article
} 


\section{Background}

Breast cancer heterogeneity makes it difficult to bring personalized medicine into the clinic. Since many years, research aimed at deciphering molecular presentation of this disease to identify subgroups of patients with clinical significance, such as prognosis or response to therapy, able to optimize patient management. The era of large-scale science, which is linked both to recent technological advances and to the availability of full genetic information, has boosted the research for new biomarkers and molecular subtyping. In 2000 and 2001, Perou and Sorlie defined five breast cancer molecular subtypes based on gene-expression profile homologies of an intrinsic gene list that included 427 unique genes: basal-like, HER2-E, luminal A, luminal B, and normal breast-like $[1,2]$. They showed that these subgroups of tumours were linked to histology, and their corresponding markers, different natural histories, response to treatment, and prognosis. Since these seminal studies, in breast cancer research, numerous gene-expression signatures (GES) with different purposes (molecular subtyping, biological pathway exploration, prognosis) emerged. Briefly, GES are composed of combinations of genes ranging from few to hundreds, which in some ways capture biological information in a non-reductionist and more informative manner. For example, researchers demonstrated that p53 GES predicted outcome better than p53 mutation status alone, and others showed that a signature of MYC activation, which better reflected MYC transcriptional output, was more informative than MYC gene expression alone $[3,4]$. In breast cancer, many studies demonstrated that GES and immunohistochemistry (IHC) were not concordant, notably for triple-negative tumours [5]. Integrated studies, combining clinico-pathological, IHC and transcriptomic data, demonstrated that GES were powerful tools to molecularly dissect breast tumours [6].

It is well recognized that breast cancer biological characteristics change as age advances. Today, there is a lack of knowledge regarding gene-expression molecular dissection of breast cancer tumours in function of age, notably in elderly patients [7]. Age-specific molecular alterations that characterize breast tumours remain to be elucidated. The vast majority of studies that aimed at exploring breast cancer in function of age are based on clinico-pathological data. Three recent works determined intrinsic molecular subtypes by means of PAM50 GES in function of age, but limited their analyses only to this subtyping GES [8-10]. In this study, we conducted functional annotation of breast cancer tumours divided in three age groups $(\leq 40,>40$ to $<70$ and $\geq 70$ years) by means of 25 GES. Available clinico-pathological data (oestrogen receptor [ER] status, HER2 status, nodal status [N], ScarffBloom-Richardson [SBR] histological grade, tumour size and evolution data) were analysed in parallel. Because of a possible ethnic bias, analyses were done twice, on a whole cohort composed of patients of mixed geographic origins, and on a Caucasian subcohort; numbers of patients were 2378 and 1413, respectively.

Functional annotation by means of 25 GES tested in this study demonstrated a decreasing aggressiveness of breast tumours in function of age based on continuous GES scoring. Furthermore, we showed that luminal B tumour of elderly patients were less aggressive than luminal B tumour of young patients.

\section{Methods}

\section{Data selection}

We exclusively looked for publicly available breast cancer Affymetrix ${ }^{\ominus}$ genomic datasets associated with clinico-pathological information, including age at diagnosis, prognosis and geographic origin of the cohorts in repositories such as Gene Expression Omnibus (GEO), ArrayExpress, author's individual web pages, and in articles, selecting those with a medium to large sample size $[11,12]$. Among other things, our study aimed at exploring molecular subtype distribution, which is known to vary in function of population origin [10, 13-16]. For this reason, we selected a Caucasian cohort from the whole population. However, precise origin of the patients was exceptionally indicated in clinico-pathological characteristics associated with genomic data. So, we selected European cohorts and three non-European ones (E_TABM_158, GSE7849 and GSE17907), for whom ethnicity was reported; non-Caucasian patients were excluded from these three studies. According to the cohorts' country of origin, we supposed that they were composed of a large majority of Caucasian women. Patients who received neoadjuvant chemotherapy and microdissected samples were not included.

\section{Data pre-processing}

Data pre-processing and normalization were described elsewhere [17] and are summarized in Additional file 1.

\section{Gene-expression signatures}

Twenty-five GES were selected for functional annotation of breast cancer tumours. Five GES were used for breast cancer molecular subtyping: PAM50, ER, molecular apocrine, basal-like and claudin-CD24. Seventeen were linked to biological processes of importance or cell types: immune response (B-cell, interferon [IFN], interleukin-8 [IL-8], MHC-1, MHC-2, T-cell, M2-macrophages/M1macrophages enrichment [M2/M1]), metabolism (adipocytes, glycolysis, iron [IRGS]) and critical biological pathways in cancer (chromosomal instability [CIN], ERBB2, HOXA, mitochondrial oxidative phosphorylation [MITO/ OXPHOS], proliferation, reactive stroma, VEGF). Finally, three prognostic GES were also used: van't Veer 70-GES, 
recurrence score (RS) and genomic grade index (GGI). Complete GES list, methods and references are briefly described in Additional file 1.

\section{Statistical analysis}

Evolution analysis based on pejorative events (local relapse, metastatic relapse or death), metastatic relapse alone (MFS) and overall survival (OS) were estimated by the Kaplan-Meier method and compared between the age groups by the Log-rank test.

Mantel-Haenszel chi-square trend test was used to analyse relations between clinico-pathological characteristics (ER, HER2, SBR histological grade, nodal status, tumour histological size) and ordered age categories. In addition, unordered multinomial logistic regression (UMLR) was used for PAM50 subtype distribution and SBR histological grade in luminal $B$ tumours in function of continuous age. Kinetics was determined by the value of odds ratio (OR). One way analysis of variance (ANOVA), followed by Tukey post-hoc test for pairwise comparisons in case of significance, was used to compare continuous variables between the three age groups. GES subtyping and scoring were done on patients for whom at least $75 \%$ of GES genes were available in their expression data (Additional file 1). Twenty four continuous GES (all GES except PAM50) score correlations were illustrated with a correlation plot along with the dendrogram corresponding to average-linkage hierarchical clustering algorithm of GES with Pearson correlation distance measure.
We considered a two-sided $p$-value of less than 0.05 to be statistically significant (for multiple comparisons, Bonferroni correction was applied); the same level was used for significance analysis of microarrays (SAM) qvalue. Mantel-Haenszel chi-square trend test, UMLR, hierarchical clustering algorithm and SAM method were done with $\mathrm{R}$ software (version 3.0.2) and packages; coin, VGAM, amap and samr, respectively. STATA ${ }^{\circ}$ was used for survival analyses (version 12.0).

\section{Results}

\section{Included patients}

We exclusively focused on Affymetrix ${ }^{\circ}$ genomic datasets to avoid cross-platform normalization issues. Fifteen publicly available datasets were pooled for a total of 2378 patients, out of whom 1413 were of Caucasian origin (Table 1). Three patients' age groups were defined: $\leq 40$ years (AG1), $>40$ to $<70$ years (AG2) and $\geq 70$ years (AG3). Numbers of patients in each age group for both populations are displayed in Tables 2 and 3.

\section{Clinico-pathological characteristics according to patients' age}

Associations with known clinico-pathological characteristics are shown in Tables 2 and 3. A significant difference was found for ER in function of age group $(p<0.0001)$ associated with a positive kinetics. This result was in line with current knowledge [33, 34]. A significant difference was found for HER2 in the Caucasian cohort $(p=0.0407)$,

Table 1 Cohorts included in our study

\begin{tabular}{|c|c|c|c|c|c|c|}
\hline$n$ & Study code & Affymetrix ${ }^{\oplus}$ array & References & Patients $\mathrm{n}$ & Geographic origins & Caucasian n \\
\hline 1 & E_TABM_158 & HG-U133A & [18] & 112 & USA & 81 \\
\hline 2 & GSE2603 & HG-U133A & [19] & 82 & USA & 0 \\
\hline 3 & GSE4922 & HG-U133A + B & [20] & 249 & Sweden & 249 \\
\hline 4 & GSE6532 & HG-U133A + B + Plus2 & [21] & 401 & UK, Sweden & 401 \\
\hline 5 & GSE7378 & HG-U133A & {$[22]$} & 54 & USA & 0 \\
\hline 6 & GSE7390 & HG-U133A & [23] & 198 & Sweden, France, UK & 198 \\
\hline 7 & GSE7849 & HG-U95A & [24] & 75 & USA & 58 \\
\hline 8 & GSE9195 & HG-U133Plus2 & {$[25]$} & 77 & UK, Sweden & 77 \\
\hline 9 & GSE16391 & HG-U133Plus2 & {$[26]$} & 55 & International & 0 \\
\hline 10 & GSE17907 & HG-U133Plus2 & {$[27]$} & 49 & France, Tunisia & 43 \\
\hline 11 & GSE19615 & HG-U133Plus2 & [28] & 115 & USA & 0 \\
\hline 12 & GSE20685 & HG-U133Plus2 & [29] & 296 & Taiwan & 0 \\
\hline 13 & GSE21653 & HG-U133Plus2 & [30] & 265 & France & 265 \\
\hline 14 & GSE25055 & HG-U133A & [31] & 309 & USA & 0 \\
\hline \multirow[t]{2}{*}{15} & GSE45255 & HG-U133A & [32] & 41 & Belgium & 41 \\
\hline & & & Total & 2378 & & 1413 \\
\hline
\end{tabular}


Table 2 Clinico-pathological characteristics according to the three patients' age groups of the whole cohort

\begin{tabular}{|c|c|c|c|c|c|}
\hline Patients' group (years and [n]) & n 2378 & $\leq 40(n=345)$ & $40<x<70(n=1667)$ & $\geq 70(n=366)$ & $p$-value \\
\hline \multicolumn{6}{|l|}{ Age (years) } \\
\hline Median (IQR) & $54(45-64)$ & $37(34-39)$ & $54(47-61)$ & $74(72-80)$ & \\
\hline \multicolumn{6}{|l|}{ ER status } \\
\hline Positive & $1671(71 \%)$ & 199 (58 \%) & $1170(71 \%)$ & $302(83 \%)$ & $<0.0001$ \\
\hline Negative & $681(29 \%)$ & $143(42 \%)$ & $477(29 \%)$ & $61(17 \%)$ & \\
\hline \multicolumn{6}{|l|}{ HER2 status } \\
\hline Positive & $136(14 \%)$ & $28(19 \%)$ & $89(13 \%)$ & 19 (17 \%) & 0.4862 \\
\hline Negative & $808(86 \%)$ & $121(81 \%)$ & $592(87 \%)$ & $95(83 \%)$ & \\
\hline \multicolumn{6}{|l|}{ Nodal status } \\
\hline Positive & $882(43 \%)$ & 130 (49\%) & $603(42 \%)$ & 149 (44 \%) & 0.4010 \\
\hline Negative & $1172(57 \%)$ & 138 (51 \%) & $847(58 \%)$ & $187(56 \%)$ & \\
\hline \multicolumn{6}{|l|}{ SBR grade } \\
\hline 1 & $302(17 \%)$ & $15(6 \%)$ & $223(17 \%)$ & $64(22 \%)$ & $<0.0001$ \\
\hline 2 & 770 (42\%) & $83(36 \%)$ & $561(44 \%)$ & $126(43 \%)$ & \\
\hline 3 & $742(41 \%)$ & $134(58 \%)$ & $504(39 \%)$ & $104(35 \%)$ & \\
\hline \multicolumn{6}{|l|}{ Tumour size } \\
\hline$\leq 20 \mathrm{~mm}$ & $626(46 \%)$ & $57(44 \%)$ & 458 (48 \%) & 111 (42\%) & 0.3993 \\
\hline$>20 \mathrm{~mm}$ & 723 (54 \%) & 72 (56 \%) & 499 (52 \%) & 152 (58 \%) & \\
\hline
\end{tabular}

Abbreviations: ER oestrogen receptor, IQR interquartile range, SBR Scarff-Bloom-Richardson histological grade

Table 3 Clinico-pathological characteristics according to the three patients' age groups of the Caucasian cohort

\begin{tabular}{|c|c|c|c|c|c|}
\hline Patients' group (years and [n]) & n 1413 & $\leq 40(n=166)$ & $40<x<70(n=974)$ & $\geq 70(n=273)$ & $p$-value \\
\hline \multicolumn{6}{|l|}{ Age (years) } \\
\hline Median (IQR) & $57(47-67)$ & $36(33-38)$ & $56(49-62)$ & $74(72-80)$ & \\
\hline \multicolumn{6}{|l|}{ ER status } \\
\hline Positive & $1054(76 \%)$ & $90(55 \%)$ & $735(76 \%)$ & $229(85 \%)$ & $<0.0001$ \\
\hline Negative & 341 (24 \%) & $73(45 \%)$ & $227(24 \%)$ & $41(15 \%)$ & \\
\hline \multicolumn{6}{|l|}{ HER2 status } \\
\hline Positive & $85(23 \%)$ & $25(36 \%)$ & 46 (19\%) & $14(22 \%)$ & 0.0407 \\
\hline Negative & 292 (77 \%) & $44(64 \%)$ & 198 (81\%) & $50(78 \%)$ & \\
\hline \multicolumn{6}{|l|}{ Nodal status } \\
\hline Positive & $498(36 \%)$ & $62(38 \%)$ & 319 (33\%) & 117 (46 \%) & 0.0263 \\
\hline Negative & 889 (64 \%) & $100(62 \%)$ & $651(67 \%)$ & $138(54 \%)$ & \\
\hline \multicolumn{6}{|l|}{ SBR grade } \\
\hline 1 & 254 (19\%) & $12(8 \%)$ & $182(20 \%)$ & $60(24 \%)$ & $<0.0001$ \\
\hline 2 & 571 (44 \%) & $57(36 \%)$ & 403 (45\%) & 111 (44\%) & \\
\hline 3 & $482(47 \%)$ & $88(56 \%)$ & $311(35 \%)$ & $83(33 \%)$ & \\
\hline \multicolumn{6}{|l|}{ Tumour size } \\
\hline$\leq 20 \mathrm{~mm}$ & 537 (49 \%) & $46(47 \%)$ & 388 (50 \%) & 103 (45\%) & 0.4794 \\
\hline$>20 \mathrm{~mm}$ & 567 (51 \%) & 52 (53\%) & 390 (50 \%) & 125 (55 \%) & \\
\hline
\end{tabular}


with a decreasing kinetics for HER2+ in function of age group. In Caucasian cohort, an increasing kinetics was found for positive nodal status $(\mathrm{N}+)$ according to age group ( $p=0.0263)$ (Table 3$)$. SBR histological grade distribution was different in function of age in the two cohorts $(p<0.0001)$ : SBR3 decreased compared with SBR1 from young to old patients in both cohorts. Histological size, dichotomized based on $20 \mathrm{~mm}$ cut-off, did not vary. However, a difference was shown when continuous size of tumour was used in whole cohort $(p=0.0337)$ and even in Caucasian one $(p=0.0354)$. Histological size was higher in AG3 compared to AG2 for Caucasian one $(p=0.0336)$. Histological size and $\mathrm{N}+$ status positive kinetics in function of age were also concordant with current knowledge [33, 35, 36].

\section{Evolution analysis}

In the whole cohort, no difference in evolution rates was observed between the three patients' age groups $(p=$ 0.1265) (Fig. 1a). Similar results were found with MFS and OS as outcomes (Additional file 2). On the contrary, in Caucasian cohort, young patients displayed a worse prognosis compared to patients of the intermediate group $(p=0.0051)$; no difference was found between elderly patients and patients of the intermediate group $(p=0.2794)$ (Fig. 1b). We have to underline that evolution analyses in
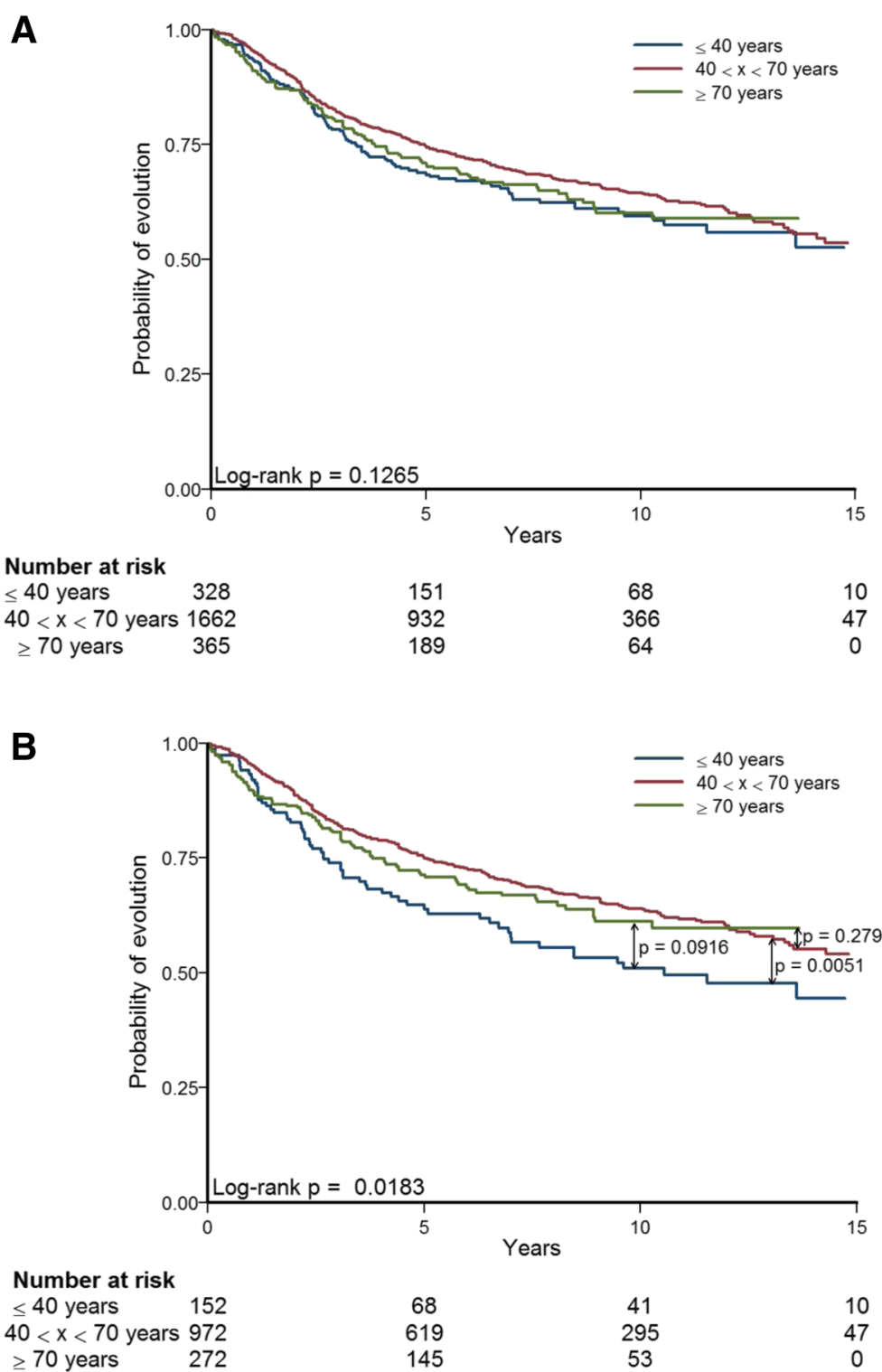

Fig. 1 Kaplan-Meier curves in function of the three patients' age groups. a Whole cohort. b Caucasian cohort 
elderly cancer patients must be interpreted cautiously because undertreatment and comorbidities in older patients distort them. This point will be discussed later.

\section{Molecular dissection by means of 25 GES}

Nineteen GES out of 24 continuous GES showed significant differences (ANOVA and Tukey post-hoc test) in function of age. ERBB2, HOXA, VEGF, Claudin-CD24 and MHC-2 GES were never significant whatever the cohort. GES molecular dissection results for the whole cohort and
Caucasian one are displayed in Table 4, Fig. 2 and Additional file 3.

A total of 2378 patients were subtyped by means of PAM50 GES. Subtype distributions are displayed in Fig. 3. Statistical analyses showed a significant growing incidence of luminal B in function of age (trend test: $p=0.0004$ and $p<0.0001$ for whole cohort and Caucasian cohort, respectively). In order to strengthen this result based on ordered age categories, we performed UMLR, which uses continuous age. UMLR result was concordant with trend test (OR $>1 ; p<0.05$ for both cohorts). Only trend test

Table 4 Continuous GES analyses interpretation in function of three age groups

\begin{tabular}{|c|c|c|c|c|c|c|c|c|c|c|c|c|}
\hline \multirow[t]{3}{*}{ GES name } & \multicolumn{6}{|c|}{ Whole cohort } & \multicolumn{6}{|c|}{ Caucasian cohort } \\
\hline & \multirow[t]{2}{*}{$p$-value } & \multirow[b]{2}{*}{1 vs 2} & \multicolumn{2}{|l|}{$p$-value } & \multicolumn{2}{|l|}{ Results } & \multirow[t]{2}{*}{$p$-value } & \multicolumn{3}{|c|}{$p$-value } & \multirow[t]{2}{*}{ Results } & \\
\hline & & & 1 vs 3 & 2 vs 3 & & & & 1 vs 2 & 1 vs 3 & 2 vs 3 & & \\
\hline \multicolumn{13}{|l|}{ Molecular subtyping } \\
\hline ER & $<0.0001$ & $<0.0001$ & $<0.0001$ & 0.0014 & $1<2<3$ & & $<0.0001$ & $<0.0001$ & $<0.0001$ & 0.0096 & $1<2<3$ & \\
\hline $\begin{array}{l}\text { Molecular- } \\
\text { apocrine }\end{array}$ & $<0.0001$ & $<0.0001$ & $<0.0001$ & 0.3376 & $1<2 \approx 3$ & & $<0.0001$ & $<0.0001$ & $<0.0001$ & 0.5479 & $1<2 \approx 3$ & \\
\hline Basal-like & $<0.0001$ & $<0.0001$ & $<0.0001$ & $<0.0001$ & $1>2>3$ & & $<0.0001$ & $<0.0001$ & $<0.0001$ & 0.0006 & $1>2>3$ & \\
\hline Claudin-CD24 & 0.8457 & & & & NS & & 0.9813 & & & & NS & \\
\hline \multicolumn{13}{|l|}{ Immune response } \\
\hline B-cell & $<0.0001$ & 0.7488 & $<0.0001$ & $<0.0001$ & $1 \approx 2>3$ & & 0.0001 & 0.9398 & 0.0053 & 0.0001 & $1 \approx 2>3$ & \\
\hline T-cell & 0.0016 & 0.7797 & 0.0667 & 0.0010 & $2>3$ & $1 \approx 2$ and $1 \approx 3$ & 0.0204 & 0.9736 & 0.1891 & 0.0153 & $2>3$ & $1 \approx 2$ and $1 \approx 3$ \\
\hline $\mathrm{MHC}-1$ & 0.0175 & 0.8578 & 0.0368 & 0.0215 & $1 \approx 2>3$ & & 0.0300 & 0.6309 & 0.0471 & 0.0589 & $1>3$ & $1 \approx 2$ and $2 \approx 3$ \\
\hline MHC-2 & 0.0133 & 0.0616 & 0.9987 & 0.0623 & NS & & 0.0486 & 0.1769 & 0.9901 & 0.1133 & NS & \\
\hline $\mathrm{M} 2 / \mathrm{M} 1$ & 0.0136 & 0.3213 & 0.0113 & 0.0616 & $1<3$ & $1 \approx 2$ and $2 \approx 3$ & 0.0429 & 0.2421 & 0.0341 & 0.2445 & $1<3$ & $1 \approx 2$ and $2 \approx 3$ \\
\hline IFN & 0.0067 & 0.3113 & 0.0059 & 0.0326 & $1 \approx 2>3$ & & 0.0633 & & & & NS & \\
\hline$\| \mathrm{L}-8$ & 0.0004 & 0.0091 & 0.0003 & 0.0917 & $1>2 \approx 3$ & & $<0.0001$ & $<0.0001$ & $<0.0001$ & 0.3518 & $1>2 \approx 3$ & \\
\hline \multicolumn{13}{|l|}{ Metabolism } \\
\hline Adipocytes & 0.0118 & 0.0140 & 0.5359 & 0.2931 & $1<2$ & $1 \approx 3$ and $2 \approx 3$ & 0.2272 & & & & NS & \\
\hline Glycolysis & 0.0044 & 0.1734 & 0.0032 & 0.0418 & $1 \approx 2>3$ & & 0.0037 & 0.0112 & 0.0031 & 0.4653 & $1>2 \approx 3$ & \\
\hline IRGS & 0.0013 & 0.0012 & 0.0047 & 0.9587 & $1>2 \approx 3$ & & 0.0122 & 0.0458 & 0.0085 & 0.3879 & $1>2 \approx 3$ & \\
\hline \multicolumn{13}{|c|}{ Critical biological pathways in cancer } \\
\hline $\mathrm{CIN}$ & $<0.0001$ & $<0.0001$ & 0.0003 & 0.9997 & $1>2 \approx 3$ & & $<0.0001$ & $<0.0001$ & 0.0012 & 0.7143 & $1>2 \approx 3$ & \\
\hline ERBB2 & 0.0771 & & & & NS & & 0.0473 & 0.9297 & 0.1280 & 0.0506 & NS & \\
\hline HOXA & 0.7895 & & & & NS & & 0.5566 & & & & NS & \\
\hline MITO/OXPHOS & $<0.0001$ & 0.2278 & $<0.0001$ & 0.0006 & $1 \approx 2<3$ & & 0.0013 & 0.8029 & 0.0097 & 0.0018 & $1 \approx 2<3$ & \\
\hline Proliferation & $<0.0001$ & $<0.0001$ & 0.0003 & 0.9989 & $1>2 \approx 3$ & & $<0.0001$ & $<0.0001$ & 0.0013 & 0.9542 & $1>2 \approx 3$ & \\
\hline Reactive stroma & $<0.0001$ & 0.1912 & 0.0487 & $<0.0001$ & $1 \approx 2>3$ & & 0.0001 & 0.1684 & 0.3841 & 0.0001 & $2>3$ & $1 \approx 2$ and $1 \approx 3$ \\
\hline VEGF & 0.4340 & & & & NS & & 0.0743 & & & & NS & \\
\hline \multicolumn{13}{|l|}{ Prognosis } \\
\hline 70-GES & $<0.0001$ & $<0.0001$ & 0.0001 & 0.9441 & $1>2 \approx 3$ & & $<0.0001$ & $<0.0001$ & $<0.0001$ & 0.9839 & $1>2 \approx 3$ & \\
\hline GGl & $<0.0001$ & $<0.0001$ & 0.0008 & 0.9303 & $1>2 \approx 3$ & & 0.0004 & 0.0003 & 0.0031 & 0.9902 & $1>2 \approx 3$ & \\
\hline RS & $<0.0001$ & $<0.0001$ & $<0.0001$ & 0.0006 & $1>2>3$ & & $<0.0001$ & $<0.0001$ & $<0.0001$ & 0.0183 & $1>2>3$ & \\
\hline
\end{tabular}

1: $\leq 40$ years; $2: 40<x<70$ years; 3: $\geq 70$ years; $E R$ oestrogen receptor, IFN interferon, IRGS iron regulatory gene signature, CIN chromosomal instability, MITO/OXPHOS mitochondrial oxidative phosphorylation, NS not significant $(p>0.05)$, 70-GES van't Veer and colleagues prognostic GES, GGl genomic grade index, $R S$ recurrence score 

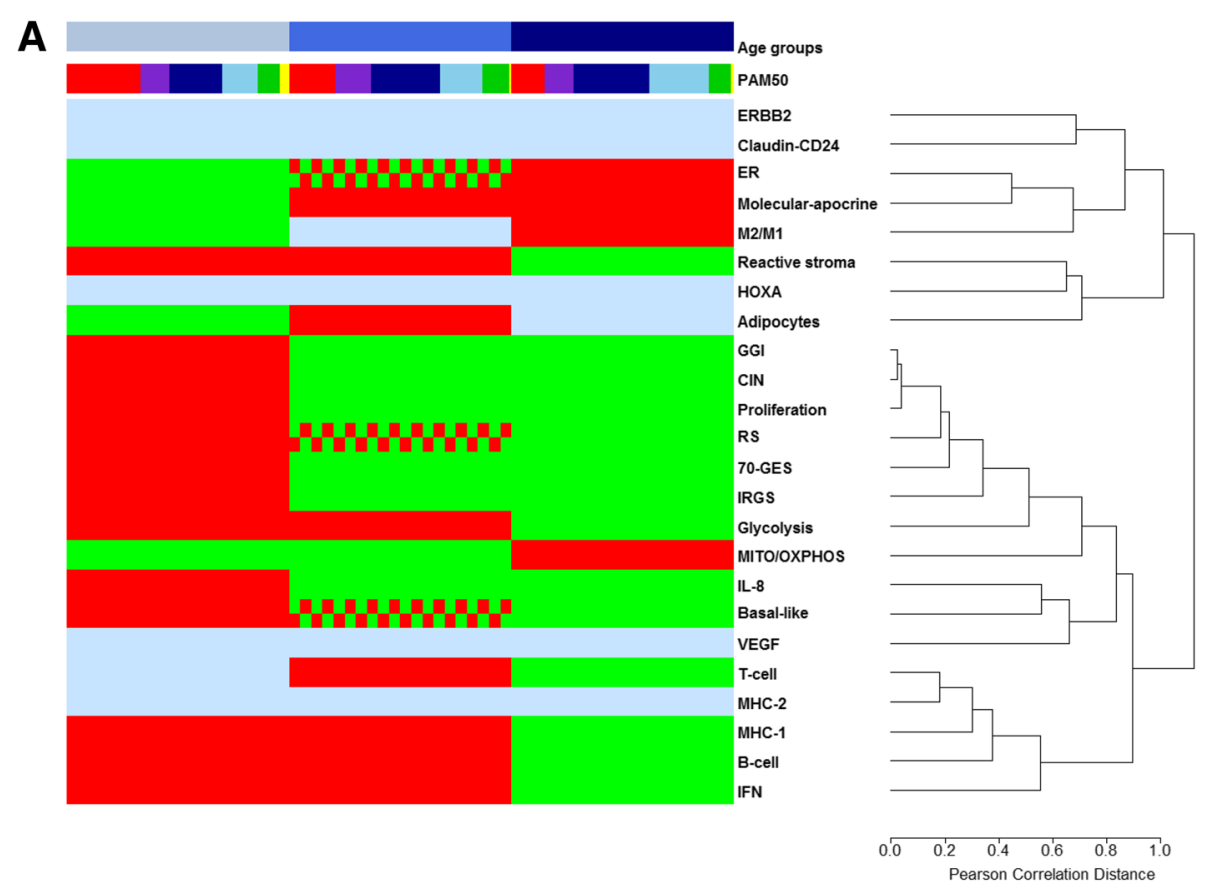

B

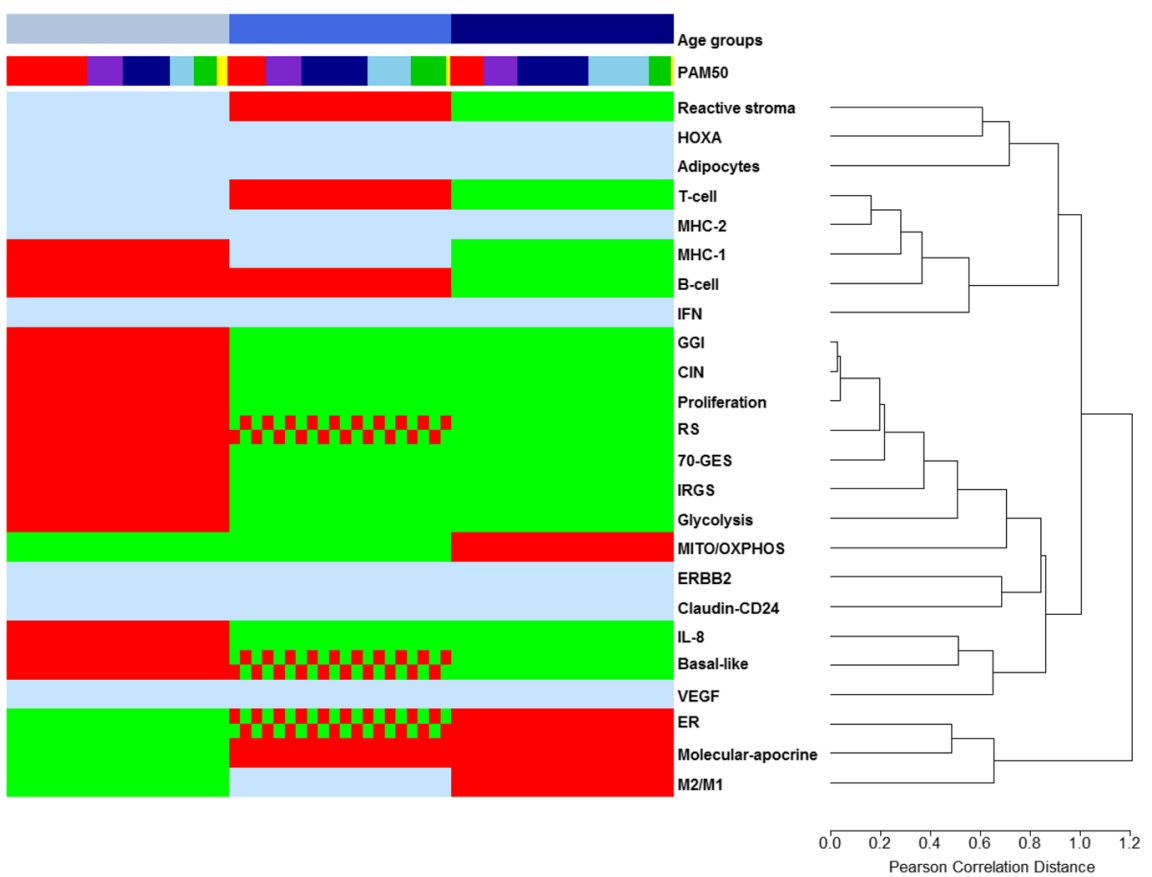

Fig. 2 Molecular dissection of breast cancer tumours in function of age. Average hierarchical clustering and heatmap showing the segregation of three age groups as a function of 24 GES scores. a Whole cohort. $\mathbf{b}$ Caucasian cohort. These figures are illustrations of table 4 statistical analyses. First row presents the three age groups: $\leq 40$ years ( $\mathbf{a}, n=345 ; \mathbf{b}, n=166)$ (sky blue), $40<x<70$ years $(\mathbf{a}, n=1667 ; \mathbf{b}, n=974)$ (medium blue) and $\geq 70$ years ( $\mathbf{a}, n=366 ; \mathbf{b}, n=273$ ) (dark blue), from left to right. Other rows, from top to bottom, present PAM50 GES subtyping (basal-like (red); HER2-E (purple); luminal A (dark blue); luminal B (sky blue); normal breast-like (green); unclassified (yellow)) and GES scores in function of age (green: low score; red and green grid pattern: intermediate score; red: high score; sky blue: not significant or not interpretable)

was significant for growing incidence of luminal A for whole cohort $(p=0.0092)$. A decreasing kinetics for basallike was found for the whole cohort (trend test: $p<0.0001$;
UMLR: OR $<1 ; p<0.0060)$ and for the Caucasian one (trend test: $p<0.0001$; UMLR: OR $<1 ; p<0.0005$ ). No other age-related kinetics was found for other subtypes. It 


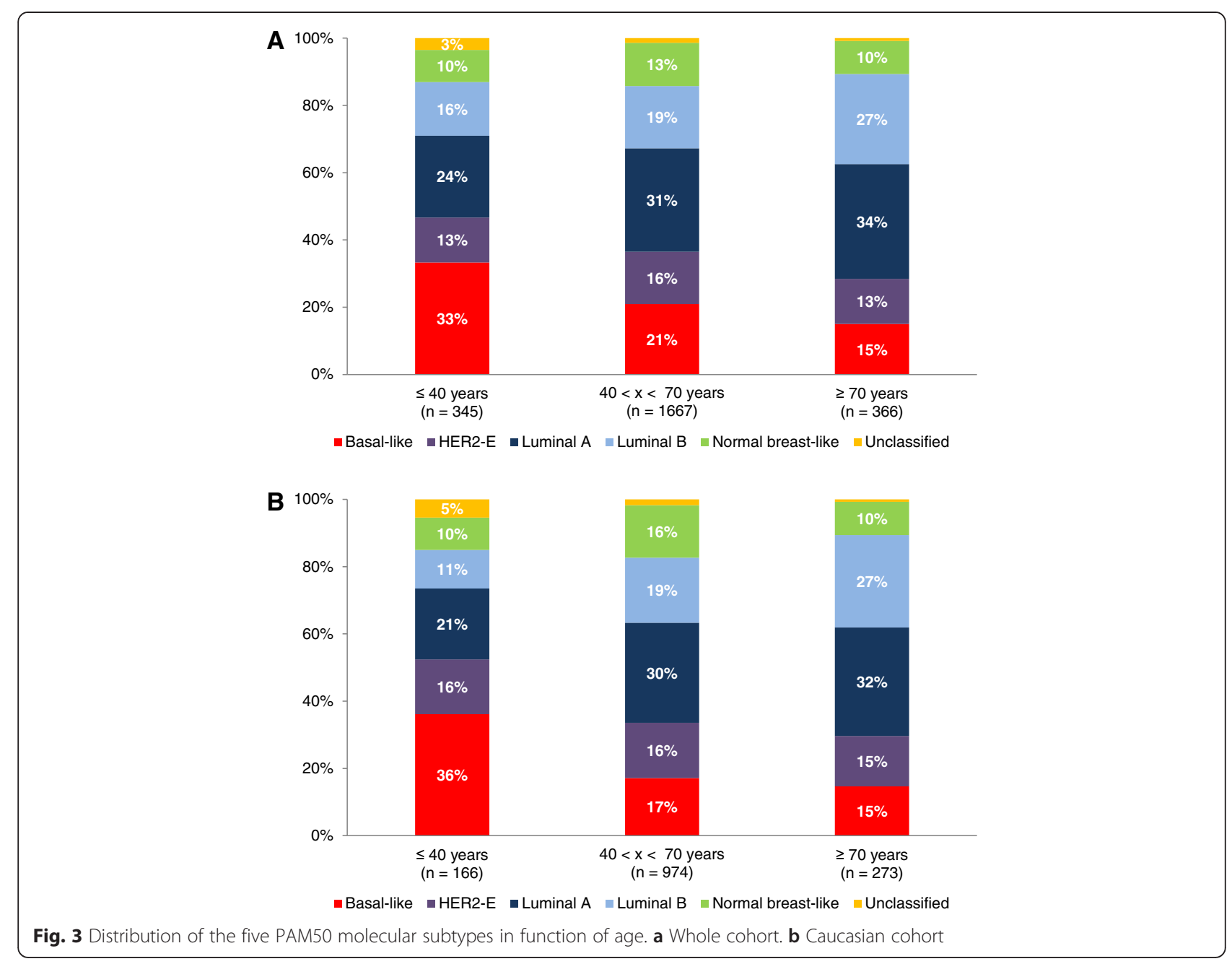

is a bit surprising to notice an increasing incidence of luminal $\mathrm{B}$, which is known to be a bad prognostic subtype, because breast cancer in advanced age has been associated with a slightly increased probability of favourable tumour biology [33]. Aggressive subtypes should be underrepresented and, on the contrary, less aggressive subtypes should be overrepresented; as we found, basal-like kinetics was in agreement with this. One hypothesis could be that luminal B tumours of elderly patients are less aggressive than those of younger patients. To explore this hypothesis, we evaluated SBR histological grade distribution in luminal B tumours in function of age (Fig. 4). We chose to conduct analysis with only SBR1 (less aggressive) and SBR3 (high aggressive) because clinical interpretation of SBR2 is complicated. Analyses showed that SBR1 percentage increased and SBR3 decreased in function of age in luminal B tumours in whole cohort (trend test: $p=0.0168$; UMLR: OR $=0.96, p=0.0035$ ) and even in Caucasian cohort (trend test: $p=0.0259$; UMLR: $\mathrm{OR}=0.95, p=0.0042$ ), which was consistent with our hypothesis. Furthermore, GES analyses of patients belonging to AG1 and AG3 showed an "AG1 > AG3" profile for reactive stroma, IRGS and RS, and an "AG1 < AG3" profile for MITO/OXPHOS, which testifies to the aggressiveness of AG1 luminal B tumours compared to AG3 luminal B tumours, in both cohorts or in the whole cohort (Additional file 4). Evolution analysis of luminal B patients in function of the three age groups did not show any difference (whole cohort: $p=0.7717$; Caucasian cohort: $p=0.3969$ ). For reasons cited above (evolution analyses biases in elderly) and discussed later, this result did not invalidate our hypothesis.

ER GES showed an increasing kinetics in function of age groups as demonstrated above by means of IHCmeasured ER. Molecular apocrine scores were the smallest in young patients and higher in the two other age groups suggesting an increased androgen receptor signalling in these last ones. This finding was concordant with some studies based on IHC, which indicated a higher rate of apocrine carcinoma in elderly and a correlation with menopausal status [37, 38]. Molecular apocrine GES scores often clustered with ER (Additional file 3). Basal-like GES scores were in concordance with basal-like PAM50 subtyping; 


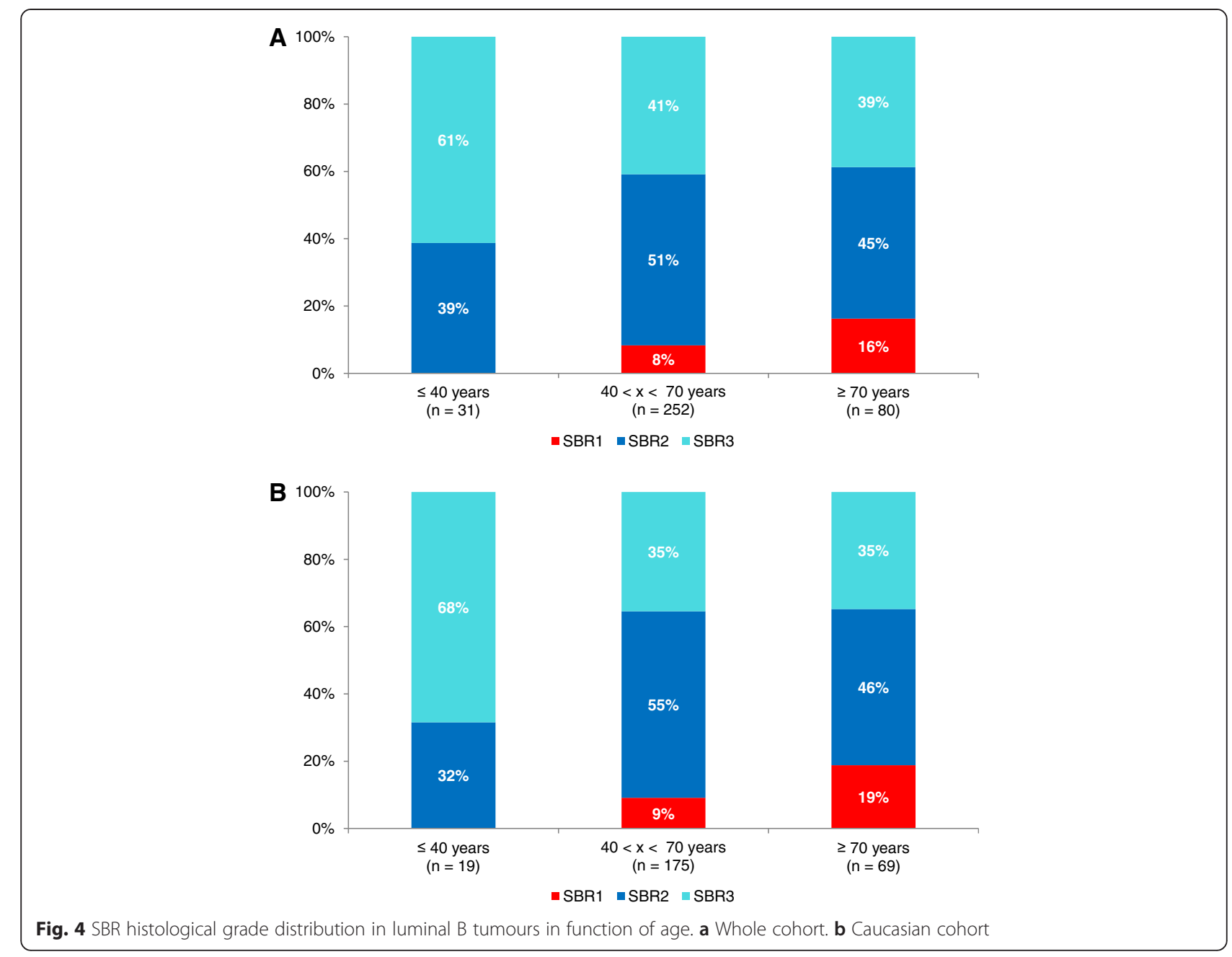

the same decreasing kinetics was found in function of age groups.

Molecular dissection of the three age groups by means of seventeen GES linked to biological processes of importance or cell types showed concordant results in both cohorts for B-cell (AG1 $\approx$ AG2 > AG3), T-cell (AG2 > AG3), IL-8, IRGS, proliferation and CIN (AG1 > AG2 $\approx$ AG3), MITO/OXPHOS (AG1 $\approx$ AG2 < AG3), and M2/M1 (AG1 $<$ AG3) (Table 4). When focusing only on the two extreme age groups, there were more concordant results between the two cohorts: basal-like, B-cell, MHC-1, IL-8, CIN, proliferation, glycolysis and IRGS displayed an "AG1 > AG3" profile, whereas ER, molecular apocrine, MITO/OXPHOS and M2/M1 displayed an "AG1 < AG3" profile.

Immune response (IR) evaluated by B-cell, MHC-1 and IL-8 GES was lower in elderly patients for both cohorts. This result could be due to immunosenescence, which was demonstrated in different transcriptomic works, rather than local low IR or local immunosuppression $[39,40]$. Here, we showed that IL-8 and basal-like always clustered together (Additional file 3). IL-8, member of the CXC chemokine family of angiogenesis/inflammation-related chemokines, is overexpressed in breast cancer, is linked to bad prognosis, has a direct role in angiogenesis and is involved in cancer stem-like cells (CSC) regulation [41, 42]. Because CSC are associated with basal-like subtype in breast cancer, IL-8/basal-like cluster might indirectly illustrate IL-8/CSC biological pathway [43]. M2/M1 showed an opposite result: high score in elderly patients (AG1 < AG3). In a recent study, which aimed at subtyping triple negative breast cancer (TNBC) tumours, we found that IR was associated with good prognosis in a basal-likeand claudin-low-enriched TNBC subtype [6]. On the contrary, M2/M1 high scores, meaning enrichment in M2 pro-tumourigenic macrophages were associated with a bad prognosis for pure basal-like TNBC subtype. M2/M1 GES applied on TNBC and PAM50 basal-like patients of our study failed to reach significance in whole cohort ( $p=$ 0.3722; $\mathrm{AG} 1=36, \mathrm{AG}=149, \mathrm{AG} 3=21$ ) and in Caucasian cohort $(p=0.3166 ; \mathrm{AG} 1=8, \mathrm{AG} 2=46, \mathrm{AG} 3=12)$. This GES needs further investigations to define its scope of use in breast cancer. 
Elderly patients had the lowest stromal reaction for the whole cohort and a lower stromal reaction compared to the intermediate age group for Caucasian one. This metagene captured different clinico-biological information. Its pattern of expression is similar to that of mammospheres and epithelial to mesenchymal signature metagene and is associated with chemoresistance [44]. A weak correlation was found for reactive stroma and adipocytes GES scores (Additional file 3).

70-GES and GGI scoring displayed an "AG1 > AG2 $\approx$ AG3" profile and RS scoring an "AG1 > AG2 > AG3" profile, respectively. In the first case, AG1 was the worst prognostic group. In the second case, a decreasing prognostic kinetics was shown, starting with AG1 as the worst prognosis age group and ending with AG3 as the best prognosis age group. RS score results might confirm the negative effect of different and less effective therapeutic management compared with younger patients on survival of elderly patients, which, despite best prognostic scores, is not significantly different from AG1 patients' survival, neither in whole cohort, nor in Caucasian one (while, in the latter cohort, AG2 patients' survival is significantly better than AG1 patients') (Fig. 1). Furthermore, 70-GES, GGI and RS clustered strongly with proliferation (Additional file 3). This can be explained by the fact that proliferation is the common driving force of these prognostic GES [45, 46].

GES analyses interpretation in function of ER status in the three age groups confirmed that proliferation (proliferation GES, 70-GES, GGI and RS) was of no value in predicting aggressiveness and prognosis in ER-negative breast cancer tumours (Additional file 5) [45].

SAM analysis identified 1116 genes with statistically significant changes in expression between the two extreme age-groups, AG1 and AG3: 432 overexpressed and 684 underexpressed genes in AG3 [47]. GO biological process enrichment analyses of these two gene lists by means of ToppGene web tool showed that cell cycle and cell migration, which are considered as basal-like hallmark, characterized AG1, and that oxidation-reduction process and lipid metabolism, which are considered as luminal hallmark, characterized AG3 (Additional file 6) [48, 49]. Although these non-hypothesis-driven results might be seen as too overly broad, they corroborated GES molecular dissection of breast tumours in function of age.

\section{Discussion}

Functional annotation may be separated into two ways of analyses: a quantitative one by means of GES, which assigns scores to patients, and a qualitative one based on gene lists, which assigns a "Gene Ontology" enrichment score to a cluster representative gene list [49].

In this work, we focused on GES scoring and used "Gene Ontology" enrichment score to corroborate our findings. Functional annotation according to the three age groups by means of clinico-pathological data was done to confirm that our cohort was representative of breast cancer population. Because of a possible ethnic bias, analyses were done twice: on the whole cohort and on the Caucasian one. Finally, there were only slight differences between whole cohort results and Caucasian results. Statistical comparisons could not be done because these cohorts were not independent.

GES used in our study can be classified into different categories: molecular subtyping $(n=5)$, immune response $(n=7)$, metabolism $(n=3)$, critical biological pathways in cancer $(n=7)$ and prognosis $(n=3)$. One question that emerged is potential biological redundancy between GES. Except for prognosis GES (70-GES, RS and GGI), CIN and PAM50, which are enriched in proliferation genes, other GES captured different biological information based on varying pathways. Numbers of genes in common between these last GES are equal or close to zero (Additional file 7). Except M2/M1 GES all other IR GES have no genes in common. However it is well known that different combination of genes can capture the same biological information. In order to look for overlapping biological information, we compared GO enrichment terms (biological process tree) linked to each GES's gene list (Fisher's exact test; $p<0.01$ ). Results are displayed in Additional files 8 and 9 . Only a few remarks can be made. In some cases, limited GES similarities exist and are concordant with biological knowledge. Biological process similarities emerged for IR GES (B-cell, IFN, IL-8, MHC-1, MHC-2 and T-cell) and as expected for GES enriched in proliferation genes (Proliferation, GGI, CIN, PAM50, RS and 70GES). Another biological process cluster is found for ER, RS, Molecular apocrine and PAM50. RS and PAM50 are strongly dependent on ER pathway. Out of 16 cancer genes included in RS GES, four belong to ESR1 cluster: ESR1, PGR, BCL2 and SCUBE2. Link between ER and molecular apocrine GES is explained by the fact that molecular apocrine subtype has a gene expression profile resembling that of ER-positive tumours [50, 51]. Our strategy might be improved by enriching GES list. Focusing on other and non-redundant critical biological pathways in cancer and increasing breast tumour transcriptome deconvolution will certainly enhance molecular dissection performance.

Molecular dissection by means of GES tested in this study confirmed that breast cancer tumours of young patients were more aggressive than tumours of older patients. Furthermore, we showed that tumour aggressiveness decreased regularly in function of age groups based on continuous GES scoring. Finally, tumours of elderly patients concentrated favourable GES scores: high ER, MITO/OXPHOS, low proliferation, basal-like, glycolysis, CIN and IRGS, and low GES prognostic score (70-GES, 
GGI and RS). These biological features were not linked to favourable disease evolution, but this result must be interpreted cautiously in elderly patients. Undertreatment in older patients with breast cancer is known to have a strong negative effect on survival [36, 52-54]. Furthermore, in our study, evolution criteria were: local relapse, metastatic relapse, or death. Due to non-cancerrelated death linked to comorbidity, which we could not identify, this last pejorative event is likely higher in elderly patients and may significantly skew evolution analyses [55]. One study reported that $44 \%$ of undertreated elderly patients died during follow-up period without disease recurrence [54].

We confirmed that age influences the incidence of molecular subtypes and found a significant growing incidence of luminal $\mathrm{B}$ and a decreasing kinetics for basallike in function of age. As we underlined, luminal B kinetics was surprising, but concordant with Jenkin's and de Kruijf's studies $[8,9]$. On the contrary, Sweeney and colleagues found an opposite result [10]. In this work, we showed that AG3 luminal B tumours were less aggressive than AG1 luminal B tumours based on SBR histological grade and four GES (IRGS, MITO/OXPHOS, reactive stroma and RS). Our results are concordant with an IHC study conducted by Morrison and colleagues [56]. They found high proliferation (Ki67 > 14 \%), high mutated P53 $(\geq 10 \%)$ and high Nottingham grade in young luminal B ( $\leq 40$ years) compared to older luminal B patients ( $\geq 50$ years). At this time, it is premature to definitively conclude and additional studies will be necessary to confirm these results.

In our study, the GES molecular dissection with the most complicated results interpretation were those provided by IR GES. An "AG1 > AG3" profile was found for B-cell, MHC-1 and IL-8, an "AG2 > AG3" for T-cell in both cohorts, and an "AG1 $\approx$ AG2 > AG3" for IFN for whole cohort. Aside from M2/M1 scores, when focusing only on the two extreme age groups, these results demonstrated a high IR in young patients and a low IR in elderly patients. Immunity is known to play a dual role in the complex interactions between tumours and the host. In established cancer, some immune cells are known to induce anti-tumoural effects (immunosurveillance) (NK cells, CD8+ T cells, Th1 cells, dendritic cells 1, M1 macrophages...), and others, pro-tumoural effects (myeloidderived suppressor cells, CD4+ T cells, Th2 cells, dendritic cells 2, M2 macrophages...) [57-59]. A dynamic model, called "immunoediting", more appropriately emphasizes the dual roles of immunity and describes tumour and immune system interactions in a chronological three phase process: elimination (immunosurveillance), dynamic equilibrium and escape $[60,61]$. The disruption of this equilibrium results from Darwinian selection of a new population of tumour clones able to escape from immune detection and/or elimination, allowing tumour progressive growth and dissemination. A second level of complexity is due to an age-dependent general decline of immune function that has been termed immunosenescence, which is associated with high circulating level of pro-inflammatory cytokines. As a consequence of these facts, most obvious, reductionist and parsimonious questions which can be asked are: In elderly patients, is low intra-tumoural IR principally due to a local effect of immunosenescence? In young patients with high intra-tumoural IR, does bad prognosis depend in part upon IR escape as proposed in immunoediting dynamic model? In answering these questions, we will be able to progress in the selection of immunotherapy strategies in function of age.

\section{Conclusions}

Our study demonstrated that a panel of GES may be used to decipher the heterogeneity of breast cancer in function of age and represented a preliminary work. A GES list including most of relevant biological pathways in breast cancer will certainly help to gain more insight into biological systems involved in this disease and will provide a framework to develop rational therapeutic strategies based on meaningful subtyping. We believe that GES are efficient tools able to dissect tumours because they do not depend on only one prototypic marker, but are composed of a combination of genes involved and/or correlated to a particular molecular pathway, and for this reason, capture more efficiently biological information.

\section{Availability of supporting data}

The datasets, listed in Table 1, used in this article are publicly available on EBI website (www.ebi.ac.uk) for E_TABM_158, and on GEO website (www.ncbi.nlm.nih. gov/geo) for all other cohorts.

\section{Additional files}

Additional file 1: GES listing, methods and references. (PDF $114 \mathrm{~kb}$ ) Additional file 2: Metastasis-free survival (MFS) and overall survival (OS) analyses. MFS: 2A1: whole cohort; 2A2: Caucasian cohort. OS: 2B1: whole cohort; 2B2: Caucasian cohort. (PDF $230 \mathrm{~kb}$ )

Additional file 3: GES scoring of breast cancer in function of age. Correlation matrices show Pearson correlation coefficients between continuous GES (red indicates a positive correlation; blue, a negative correlation) and dendrograms show mutual relationships of all signatures (scores were used for average-link hierarchical clustering using the Pearson correlation as a distance metric). 2A1: AG1, whole cohort; 2A2: AG2, whole cohort; 2A3: AG3 whole cohort; 2B1: AG1, Caucasian cohort; 2B2: AG2, Caucasian cohort; 2B3: AG3, Caucasian cohort. (PDF 1689 kb)

Additional file 4: GES analyses differentiating AG1 and AG3 luminal B patients. $A G 1>A G 3$ means that $A G 1$ GES score is superior to $A G 3$ GES score, and inversely. (PDF $57 \mathrm{~kb}$ )

Additional file 5: Continuous GES analyses interpretation in function of ER status in the three age groups. (PDF $75 \mathrm{~kb}$ ) 
Additional file 6: GO biological process enrichment analyses of most differentially expressed genes between AG1 and AG3. Head of columns represents SAM gene lists used as ToppGene inputs. The ten first significant biological processes are displayed (cutoff $p$-value $=0.01$ ). (PDF $45 \mathrm{~kb}$ )

Additional file 7: Number of genes in common between GES. (PDF $46 \mathrm{~kb}$ )

Additional file 8: Number of GO enrichment terms (biological tree) in common between GES's gene lists. (PDF $68 \mathrm{~kb}$ )

Additional file 9: Percentage of GO enrichment terms (biological tree) in common between GES's gene lists, sorted in decreasing order. (PDF $99 \mathrm{~kb}$ )

\section{Abbreviations}

70-GES: van't Veer prognostic GES; AG1: age group $n^{\circ} 1$ ( $\leq 40$ years); AG2: age group $n^{\circ} 2$ ( $>40$ to $<70$ years); AG3: age group $n^{\circ} 3$ ( $\geq 70$ years); ANOVA: analysis of variance; CIN: chromosomal instability; CSC: cancer stem-like cells; ER: oestrogen receptor (+: positive); GES: gene-expression signature; GGl: genomic grade index; IFN: interferon; IHC: immunohistochemistry; IR: immune response; IRGS: iron regulatory gene signature; IL-8: interleukin-8; MITO/OXPHOS: mitochondrial oxidative phosphorylation; M2-macrophages/ M1-macrophages enrichment; N: nodal status (+: positive); OR: odds ratio; PAM: prediction analysis for microarrays; RS: recurrence score; SAM: significance analysis of microarrays; SBR: Scarff-Bloom-Richardson histological grade; TNBC: triple negative breast cancer; UMLR: unordered multinomial logistic regression; VEGF: vascular endothelial growth factor.

\section{Competing interests}

The authors declare that they have no competing interests.

\section{Author's contributions}

PJ, ZS and MC conceived and designed the study. WG performed bioinformatics. HL, CGC, LC and SC carried out statistical analyses. PJ, WG, ZS and MC selected patient clinico-pathological data. PJ, ZS and MC interpreted data. All authors have been involved in drafting the manuscript. All authors read and approved the final manuscript.

\section{Acknowledgements}

We acknowledge UNICANCER (Fédération Nationale des Centres de Lutte contre le Cancer, France), which supplied statistician salary (Hamza Lasla).

\section{Author details}

${ }^{1}$ Bioinfomics unit, Integrated Centre for Oncology - René Gauducheau, Bd J. Monod, Nantes, Saint Herblain Cedex 44805, France. ${ }^{2}$ Cancer Genomic Unit, Integrated Centre for Oncology - René Gauducheau, Bd J. Monod, Nantes, Saint Herblain Cedex 44805, France. ${ }^{3}$ INSERM U892, IRT-UN, 8 quai Moncousu, Nantes Cedex 44007, France. ${ }^{4}$ Départemental de Vendée - site de Montaigu, Polyvalent medicine service, Centre Hospitalier, 54, rue Saint Jacques, BP 259, Montaigu 85602, France. ${ }^{5}$ Biostatistics unit, Integrated Centre for Oncology - René Gauducheau, Bd J. Monod, Nantes, Saint Herblain Cedex 44805, France. ${ }^{6}$ Mathematics laboratory, UMR CNRS 6623 et Université de Franche Comté, 16 route de Gray, Besançon Cedex 25030, France. ${ }^{7}$ Medical oncology service, Integrated Centre for Oncology - René Gauducheau, Bd J. Monod, Nantes, Saint Herblain Cedex 44805, France. ${ }^{8}$ Biopatholgy department, Integrated Centre for Oncology - René Gauducheau, Bd J. Monod, Nantes, Saint Herblain Cedex 44805, France.

Received: 12 June 2015 Accepted: 17 November 2015

Published online: 23 November 2015

\section{References}

1. Perou CM, Sorlie T, Eisen MB, van de Rijn M, Jeffrey SS, Rees CA, et al. Molecular portraits of human breast tumours. Nature. 2000;406:747-52.

2. Sorlie T, Perou CM, Tibshirani R, Aas T, Geisler S, Johnsen H, et al. Gene expression patterns of breast carcinomas distinguish tumor subclasses with clinical implications. Proc Natl Acad Sci USA. 2001;98:10869-74.

3. Miller LD, Smeds J, George J, Vega VB, Vergara L, Ploner A, et al. An expression signature for $\mathrm{p} 53$ status in human breast cancer predicts mutations status, transcriptional effects, and patients survival. Proc Natl Acad Sci USA. 2005;102:13550-5.

4. Miller TW, Balko JM, Ghazoui Z, Dunbier A, Anderson H, Dowsett M, et al A gene expression signature from human breast cancer cells with acquired hormone independence identifies MYC as a mediator of antiestrogen resistance. Clin Cancer Res. 2011;17:2024-34.

5. Prat A, Perou CM. Deconstructing the molecular portraits of breast cancer. Mol Oncol. 2011:5:5-23.

6. Jézéquel $P$, Loussouarn $D$, Guérin-Charbonnel C, Campion L, Vanier $A$, Gouraud W, et al. Gene-expression molecular subtyping of triple-negative breast cancer tumours: importance of immune response. Breast Cancer Res. 2015;17:43.

7. Thomas GA, Leonard RCF. How age affects the biology of breast cancer. Clin Oncol. 2009;21:81-5.

8. de Kruijf E, Bastiaannet E, Rubertá F, de Craen AJ, Kuppen PJ, Smit VT, et al. Comparison of frequencies and prognostic effect of molecular subtypes between young and elderly breast cancer patients. Mol Oncol. 2014;8:1014-25.

9. Jenkins EO, Deal AM, Anders CK, Prat A, Perou CM, Carey LA, et al. Age-specific changes in intrinsic breast cancer subtypes. Oncologist. 2014;19:1076-83.

10. Sweeney C, Bernard PS, Factor RE, Kwan ML, Habel LA, Quesenberry Jr CP, et al. Intrinsic subtypes from PAM50 gene expression assay in a populationbased breast cancer cohort: differences by age, race, and tumor characteristics. Cancer Epidemiol Biomarkers Prev. 2014;23:714-24.

11. Edgar R, Domrachev M, Lash AE. Gene Expression Omnibus: NCBI gene expression and hybridization array data repository. Nucleic Acids Res. 2002;30:207-10.

12. Hubble J, Demeter J, Jin H, Mao M, Nitzberg M, Reddy TB, et al. Implementation of GenePattern within the Stanford Microarray Database. Nucleic Acids Res. 2009;37(Database Issue):D898-901.

13. Carey LA, Perou CM, Livasy CA, Dressler LG, Cowan D, Conway K, et al. Race, breast cancer subtypes, and survival in the Carolina breast cancer study. JAMA. 2006;295:2492-502.

14. Su Y, Zheng Y, Zheng W, Gu K, Chen Z, Li G, et al. Distinct distribution and prognostic significance of molecular subtypes of breast cancer in Chinese women: a population-based cohort study. BMC Cancer. 2011;11:292.

15. Preat F, Simon $P$, Noel JC. Differences in breast carcinoma immunohistochemical subtypes between immigrant Arab and European women. Diagn Pathol. 2014;9:26.

16. Singh M, Ding Y, Zhang LY, Song D, Gong Y, Adams S, et al. Distinct breast cancer subtypes in women with early-onset disease across races. Am J Cancer Res. 2014;4:337-52.

17. Jézéquel $P$, Campone M, Gouraud W, Guérin-Charbonnel C, Leux C, Ricolleau $G$, et al. bc-GenExMiner: an easy-to-use online platform for gene prognostic analyses in breast cancer. Breast Cancer Res Treat. 2012;131:765-75.

18. Chin K, DeVries S, Fridlyand J, Spellman PT, Roydasgupta R, Kuo WL, et al. Genomic and transcriptional aberrations linked to breast cancer pathophysiologies. Cancer Cell. 2006;10:529-41.

19. Minn AJ, Gupta GP, Siegel PM, Bos PD, Shu W, Giri DD, et al. Genes that mediate breast cancer metastasis to lung. Nature. 2005;436:518-24.

20. Ivshina AV, George J, Senko O, Mow B, Putti TC, Smeds J, et al. Genetic reclassification of histologic grade delineates new clinical subtypes of breast cancer. Cancer Res. 2006;66:10292-301.

21. Loi S, Haibe-Kains B, Desmedt C, Lallemand F, Tutt AM, Gillet C, et al. Definition of clinically distinct molecular subtypes in estrogen receptorpositive breast carcinomas. J Clin Oncol. 2007;25:1239-46.

22. Zhou Y, Yau C, Gray JW, Chew K, Dairkee SH, Moore DH, et al. Enhanced NF kappa B and AP-1 transcriptional activity associated with antiestrogen resistant breast cancer. BMC Cancer. 2007;7:59.

23. Desmedt C, Piette F, Loi S, Wang Y, Lallemand F, Haibe-Kains B, et al. Strong time dependence of the 76-gene prognostic for node-negative breast cancer patients in the TRANSBIG multicenter independent validation series. Clin Cancer Res. 2007;13:3207-14

24. Anders CK, Acharya CR, Hsu DS, Broadwater G, Garman K, Foekens JA, et al. Age-specific differences in oncogenic pathway deregulation seen in human breast tmors. PLoS One. 2008;2, e1373.

25. Loi S, Haibe-Kains B, Desmedt C, Wirapati P, Lallemand F, Tutt AM, et al. Predicting prognosis using molecular profiling in estrogen receptor-positive breast cancer treated with tamoxifen. BMC Genomics. 2008;9:239.

26. Desmedt C, Giobbie-Hurder A, Neven P, Paridaens R, Christiaens MR, Smeets A, et al. The gene expression grade index: a potential predictor of relapse for 
endocrine-treated breast cancer patients in the BIG 1-98 trial. BMC Med Genomics. 2009;2:40.

27. Sircoulomb F, Bekhouche I, Finetti P, Adélaïde J, Ben Hamida A, Bonansea J, et al. Genome profiling of ERBB2-amplified breast cancers. BMC Cancer. 2010;10:539

28. Li Y, Zou L, Li Q, Haibe-Kains B, Tian R, Li Y, et al. Amplification of LAPTM4B and YWHAZ contributes to chemotherapy resistance and recurrence of breast cancer. Nat Med. 2010;16:214-8.

29. Kao KJ, Chang KM, Hsu HC, Huang AT. Correlation of microarray-based breast cancer molecular subtypes and clinical outcomes: implications for treatment optimization. BMC Cancer. 2011;11:143.

30. Sabatier R, Finetti P, Cervera N, Lambaudie E, Esterni B, Mamessier E, et al. A gene expression signature identifies two prognostic subgroups of basal breast cancer. Breast Cancer Res Treat. 2011;126:407-20.

31. Hatzis C, Pusztai L, Valero V, Booser DJ, Esserman L, Lluch A, et al. A genomic predictor of response and survival following taxane-anthracycline chemotherapy for invasive breast cancer. JAMA. 2011;305:1873-81.

32. Nagalla S, Chou JW, Willingham MC, Ruiz J, Vaughn JP, Dubey P, et al. Interactions between immunity, proliferation and molecular subtype in breast cancer prognosis. Genome Biol. 2013;14:R34

33. Diab SG, Elledge RM, Clark GM. Tumor characteristics and clinical outcome of elderly women with breast cancer. J Natl Cancer Instit. 2000;92:550-6.

34. Dunnwald LK Rossing MA, Li Cl. Hormone receptor status, tumor characteristics, and prognosis: a prospective cohort of breast cancer patients. Breast Cancer Res. 2007;9:R6

35. Wildiers H, Van Calster B, van de Poll-Franse LV, Hendrickx W, Røislien J, Smeets $A$, et al. Relationship between age and axillary lymph node involvement in women with breast cancer. J Clin Oncol. 2009;27:2931-7.

36. Schonberg MA, Marcantonio ER, Li D, Silliman RA, Ngo L, McCarthy EP. Breast cancer among the oldest old: tumor characteristics, treatment choices, and survival. J Clin Oncol. 2010;28:2038-45.

37. Honma N, Sakamoto G, Akiyama F, Esaki Y, Sawabe M, Arai T, et al. Breast carcinoma in women over the age of 85: distinct histological pattern and androgen, oestrogen, and progesterone receptor status. Histopathology. 2003:42:120-7.

38. Agoff S, Swanson P, Linden H, Hawes S, Lawton T. Androgen receptor expression in estrogen receptor-negative breast cancer. Immunohistochemical, clinical, and prognostic associations. Am J Clin Pathol. 2003;120:725-31.

39. Cao JN, Gollapudi S, Sharman EH, Jia Z, Gupta S. Age-related alterations of gene expression patterns in human CD8+ T cells. Aging Cell. 2010;9:19-31

40. Harries LW, Hernandez D, Henley W, Wood AR, Holly AC, Bradley-Smith RM, et al. Human aging is characterized by focused changes in gene expression and deregulation of alternative splicing. Aging Cell. 2011;10:868-78.

41. Todorovic-Rkovic N, Milovanovic J. Interleukin-8 in breast cancer progression. J Interferon Cytokine Res. 2013;33:563-70.

42. Singh J, Simoes B, Howell SJ, Farnie G, Clarke R. Recent advances reveal IL-8 signaling as a potential key to targeting breast cancer stem cells. Breast Cancer Res. 2013;15:210.

43. Honeth G, Bendahl PO, Ringnér M, Saal LH, Gruvberger-Saal SK, Lövgren K, et al. The CD44+/CD24- phenotype is enriched in basal-like breast tumors. Breast Cancer Res. 2008;10:R53.

44. Farmer $\mathrm{P}$, Bonnefoi $\mathrm{H}$, Anderle $\mathrm{P}$, Cameron D, Wirapati $\mathrm{P}$, Becette $\mathrm{V}$, et al. A stroma-related gene signature predicts resistance to neoadjuvant chemotherapy in breast cancer. Nat Med. 2009;15:68-74.

45. Desmedt C, Haibe-Kains B, Wirapati P, Buyse M, Larsimont D, Bontempi G, et al. Biological processes associated with breast cancer clinical outcome depend on the molecular subtypes. Clin Cancer Res. 2008;14:5158-65.

46. Wirapati P, Sotiriou C, Kunkel S, Farmer P, Pradervand S, Haibe-Kains B, et al. Meta-analysis of gene expression profiles in breast cancer: toward a unified understanding of breast cancer subtyping and prognosis signatures. Breast Cancer Res. 2008;10:R65.

47. Tusher V, Tibshirani R, Chu G. Significance analysis of microarrays applied to transcriptional responses to ionizing radiation. Proc Natl Acad Sci USA 2001;98:5116-21.

48. Ashburner M, Ball CA, Blake JA, Botstein D, Butler H, Cherry JM, et al. Gene ontology: tool for the unification of biology. The Gene Ontology Consortium. Nat Genet. 2000;25:25-9.

49. Chen J, Bardes EE, Aronow BJ, Jegga AG. ToppGene Suite for gene list enrichment analysis and candidate gene prioritization. Nucleic Acids Res. 2009:37:W305-11.
50. Farmer $\mathrm{P}$, Bonnefoi $\mathrm{H}$, Becette $\mathrm{V}$, Tubiana-Hulin M, Fumoleau $\mathrm{P}$, Larsimont $\mathrm{D}$, et al. Identification of molecular apocrine breast tumours by microarray analysis. Oncogene. 2005;24:4660-71.

51. Doane AS, Danso M, Lal P, Donaton M, Zhang L, Hudis C, et al. An estrogen receptor-negative breast cancer subset characterized by a hormonally regulated transcriptional program and response to androgen. Oncogene. 2006;25:3994-4008

52. Muss HB, Woolf S, Berry D, Cirrincione C, Weiss RB, Budman D, et al. Adjuvant chemotherapy in older and younger women with lymph $<$ ?show [?A3B2 show \$9\#?]?>node-positive breast cancer. JAMA. 2005;293:1073-81.

53. Biganzoli L, Wildiers H, Oakman C, Marotti L, Loibl S, Kunkler I, et al. Management of elderly patients with breast cancer: updated recommendations of the international society of geriatric oncology (SIOG) and European society of breast cancer specialists (EUSOMA). Lancet Oncol. 2012;13:e148-160.

54. Rocco N, Rispoli C, Pagano G, Ascione S, Compagna R, Danzi M, et al. Undertreatment of breast cancer in the elderly. BMC Surg. 2013;13:S26.

55. Satariano WA, Ragland DR. The effect of comorbidity on 3-year survival of women with primary breast cancer. Ann Intern Med. 1994;120:104-10.

56. Morrison DH, Rahardja D, King E, Peng Y, Sarode VR. Tumour biomarker expression relative to age and molecular subtypes of invasive breast cancer. Br J Cancer. 2012;107:382-7.

57. Finak G, Bertos N, Pepin F, Sadekova S, Souleimanova M, Zhao H, et al. Stromal gene expression predicts clinical outcome in breast cancer. Nat Med. 2008;14:518-27.

58. Salgado R, Denkert C, Demaria S, Sirtaine N, Klauschen F, Pruneri G, et al. The evaluation of tumor-infiltrating lymphocytes (TILS) in breast cancer: recommendations by an international TILs-working group 2014. Ann Oncol. 2015;26:259-71.

59. Hagerling C, Casbon AJ, Werb Z. Balancing the innate immune system in tumor development. Trends Cell Biol. 2015;25:214-20.

60. Dunn GP, Old L, Schreiber RD. The three Es of cancer immunoediting Annu Rev Immunol. 2004:22:329-60.

61. Liu K, Caldwell SA, Abrams SI. Immune selection and emergence of aggressive tumor variants as negative consequences of Fas-mediated cytotoxicity and altered IFN- $\gamma$-regulated gene expression. Cancer Res. 2005;65:4376-88

\section{Submit your next manuscript to BioMed Central and we will help you at every step:}

- We accept pre-submission inquiries

- Our selector tool helps you to find the most relevant journal

- We provide round the clock customer support

- Convenient online submission

- Thorough peer review

- Inclusion in PubMed and all major indexing services

- Maximum visibility for your research

Submit your manuscript at www.biomedcentral.com/submit 